\title{
Examination of Effective Body Sway Parameters for Healthy Elderly
}

\author{
Tamotsu Kitabayashi ${ }^{1}$, Shinichi Demura ${ }^{2}$, Hiroki Aoki ${ }^{3{ }^{* *}}$ \\ ${ }^{1}$ Tokyo University of Science, Japan \\ ${ }^{2}$ Kanazawa University, Ishikawa, Japan \\ ${ }^{3}$ National Institute of Technology, Fukui College, Fukui, Japan \\ *Corresponding author: aoki@fukui-nct.ac.jp
}

\begin{abstract}
This study aimed to examine body sway characteristics and to propose effective body sway parameters for healthy elderly. The subjects were 311 healthy elderly and 380 healthy young adults. None of the subjects had evidence or known history of gait, posture, or skeletal disorders. The center of foot pressure measurement for 1 min was performed in 1 trial Using Anima's stabilometer G5500. The data sampling frequency was $20 \mathrm{~Hz}$. Thirty parameters with high reliability were selected from 5 domains of distance, area, velocity, power spectrum, and body sway vector. In comparison with the results for young adults, the elderly had markedly larger values of mean path length and root mean square of $\mathrm{x}$-axis, as well as for 5 velocity parameters and 4 vector velocity parameters. In addition, approximately $10 \%$ of the data for parameters related to body sway velocity, especially front/back body sway, even in the healthy elderly fell in an abnormal range of values (mean $\pm 3 \mathrm{SD}$ ). In conclusion, the following parameters may be useful to adequately and simply evaluate the body sway characteristics of the elderly: sway size, sway velocity, and from a directional viewpoint, sway size in the right/left direction and sway velocity in the front/back direction.
\end{abstract}

Keywords: aging, center of foot pressure, static balance

Cite This Article: Tamotsu Kitabayashi, Shinichi Demura, and Hiroki Aoki, "Examination of Effective Body Sway Parameters for Healthy Elderly.” American Journal of Sports Science and Medicine, vol. 6, no. 1 (2018): 22-27. doi: 10.12691/ajssm-6-1-5.

\section{Introduction}

Body sway during a static upright posture is controlled by the synkinesis of the limbs and body trunk, based on information from posture adjustment functions such as the visuosensory, vestibular, and proprioception organ systems [1] (Cernacek et al., 1973). The important brain and nerve system function of holding stable posture is maintained until a relatively old age after sufficient development (Okada et al., 2001). However, it is very difficult for the elderly to maintain an upright posture because of the marked decline in the above functions at an advanced age. Cummings et al. (2000) reported that the elderly have more co-operative disorders of the input and output systems used for posture maintenance. When examining the upright posture of elderly individuals, it is necessary to use effective parameters that can adequately evaluate their body sway characteristics.

The Japan Society for Equilibrium Research recommends area, locus length, displacement, power spectrum, position and velocity vectors, and amplitude probability density distribution as evaluation items of their body sway test. Perimeter area and total locus length are used to evaluate sway size, and unit time locus length is used to evaluate sway velocity. The area surrounding the mean path length (total locus length/perimeter area) is useful for evaluating the details of posture control. Right/left displacement is the right/left distance of the center of foot pressure (COP) and the body sway average center, and front/back displacement is the corresponding front/back distance. Right/left displacement can be used to evaluate labyrinthine deviation induced by unilateral labyrinthine dysfunction. Front/back displacement can be used to evaluate bilateral labyrinthine dysfunction, Parkinson's disease, and hypertonia of antigravity muscle or hypofunction by cerebellar dysfunction. A position vector is the vector from COP to a sampling point by the sum for all 8 directions. This vector can be used to evaluate the spread of body sway. These parameters are effective because unilateral labyrinthine dysfunction shows considerable unusual right/left body sway induced by labyrinthine deviation in the acute phase. A velocity vector is also divided in the 8 directions. These parameters can be used to evaluate the directivity of body sway and are effective because unilateral labyrinthine dysfunction shows large right/left body sway in the acute phase and front/back body sway in the chronic phase.

Watanabe et al. (2009) examined the relationship between the muscle mass of psoas major muscle and body sway using the total length parameter and the 
circumference area parameter for the elderly. Hirase et al. [2] also examined the relationship between leg strength and body sway using the same parameters for the elderly. In addition, Tanida et al. (2011) examined the change in balance function in exercise therapy in local elderly people using multilateral parameters such as total length, mean path length, front/back length, right/left length, circumference area, front/back maximum amplitude, and right/left maximum amplitude.

As described above, the parameters used to evaluate body sway in the elderly vary considerably, i.e., they have not been unified. Therefore, it is necessary to examine effective body sway parameters. This study aimed to examine body sway characteristics and to propose effective body sway parameters for healthy elderly.

\section{Methods}

\subsection{Subjects}

The subjects were 311 healthy elderly (age: $71.2 \pm 6.27$ years, height: $161.5 \pm 6.57 \mathrm{~cm}$, weight: $60.1 \pm 8.29 \mathrm{~kg}$ ) and 380 healthy young adults (age: $24.3 \pm 2.02$ years, height: $173.3 \pm 5.55 \mathrm{~cm}$, weight: $67.1 \pm 7.01 \mathrm{~kg}$ ). None of the subjects had evidence or known history of gait, posture, or skeletal disorders. Informed consent was obtained from all subjects. The experimental protocol was approved by the Ethics Committee on Human Experimentation of the Faculty of Human Science, Kanazawa University (No.2012-03).

\subsection{Experimental Procedure}

The measurement procedure followed the method prescribed in the standardization of the stabilometry test (Japan Society for Equilibrium Research, 1984). The subjects maintained a static upright posture with closed feet (Romberg posture) for 1 min. During the testing, they were instructed to watch a circular target placed at the eye level and stood barefoot with their arms held comfortably and their eyes open. The measurements began after the subject's posture and eyes were stable. Measurements were performed in 1 trial.

\subsection{Experimental Instrument}

The measurement device used was Anima's stabilometer G5500. This device can calculate COP of vertical loads from the values of 3 vertical load sensors, which are located in the corners of an isosceles triangle on a level surface. The data sampling frequency was $20 \mathrm{~Hz}$.

\subsection{Parameters}

Thirty parameters with high reliability were selected from the following 5 domains: distance represented sway size (4 parameters), area represented sway magnitude (3 parameters), velocity represented sway velocity (5 parameters), power spectrum represented sway frequency (5 position parameters and 5 velocity parameters), and vector represented sway direction (4 position parameters and 4 velocity parameters) [3]. In addition, this study did not correct parameters according to physique because Kitabayashi et al. [4] reported that the influence of physique on body sway parameters is small $(r<0.4)$; this conclusion was also confirmed in the present study.

\subsection{Statistical Analysis}

The t-test was used to examine the mean difference between the elderly and the young adults for COP parameters. The effect size (ES) was calculated to examine the size of the mean difference. An ES of $\leq 0.2$ is generally interpreted as a small difference, and $\geq 0.8$ as a large difference. Relationships among parameters were examined using Pearson's correlations coefficient. Their significant difference in both groups was tested. The statistical significance $(\alpha)$ level was set at $p<0.05$. $\alpha$ was controlled on the basis of Bonferroni's method.

\section{Results}

Table 1 shows the test results for the mean differences between the young adults and the elderly and ES for COP parameters. Significant differences were found in 25 parameters and not in 5 others related to the power spectrum. The elderly had markedly larger values of mean path length and root mean square of the x-axis, as well as for 5 velocity parameters and 4 vector velocity parameters (ES = 0.71-1.00).

Table 2 and Table 3 show correlations among the parameters in the young adults and the elderly, respectively, and the test results for the homogeneity of correlations. Most correlations were significant (gray). In both groups, strong correlations $(r>0.8)$ were found between the mean path length and parameters related to velocity and vector velocity, between the root mean square and area parameters, and between velocity, vector velocity parameters, and related parameters. Correlations between the parameters of distance and area and $y$ direction parameters in the young adults were significantly greater than those in the elderly (* in Table 2 ). On the other hand, correlations between distance parameters and power spectrum parameters, between velocity parameters and power spectrum parameters, and between power spectrum parameters and vector velocity parameters in the elderly were significantly greater than those in the young adults (* in Table 3$)$.

Table 4 shows the frequency at which parameters fell outside the mean $\pm 1-3$ SD range for the young adults. More than $10 \%$ of measurements for 29 parameters, excluding the power spectrum of r-axis, fell outside the mean \pm 1 SD range, similar to approximately $50 \%$ of measurements of mean path length, velocity parameters, and vector velocity parameters. Many parameters $(<10 \%)$ in the elderly fell within the mean \pm 3 SD range for the young adults, but mean path length, 3 velocity parameters (y direction), and 3 vector velocity parameters (y direction) were $\geq 10 \%$ outside this range. 
Table 1. Significant differences between means of the young adults and the elderly, and ES for sway parameters

\begin{tabular}{|c|c|c|c|c|c|c|c|}
\hline & \multicolumn{2}{|c|}{ young adults } & \multicolumn{2}{|c|}{ elderly } & \multirow[b]{2}{*}{$\mathrm{t}$} & & \multirow[b]{2}{*}{$\mathrm{ES}$} \\
\hline & $\mathrm{M}$ & $S D$ & $M$ & SD & & & \\
\hline Mean path length $(\mathrm{cm} / \mathrm{sec})$ & 1.21 & 0.41 & 1.75 & 0.78 & 11.75 & $*$ & 0.90 \\
\hline Root mean square $(\mathrm{cm})$ & 0.72 & 0.24 & 0.83 & 0.22 & 6.14 & * & 0.47 \\
\hline Root mean square of $X$-axis $(\mathrm{cm})$ & 0.44 & 0.15 & 0.55 & 0.16 & 9.24 & $*$ & 0.71 \\
\hline Root mean square of $Y$-axis $(\mathrm{cm})$ & 0.56 & 0.22 & 0.61 & 0.20 & 3.15 & * & 0.24 \\
\hline Area surrounding mean path length $(1 / \mathrm{cm})$ & 26.88 & 10.06 & 24.47 & 9.08 & 3.26 & $*$ & 0.25 \\
\hline Area surrounding maximal amplitude rectangular $\left(\mathrm{cm}^{2}\right)$ & 7.23 & 5.44 & 10.97 & 6.28 & 8.36 & $*$ & 0.64 \\
\hline Area surrounding root mean square $(\mathrm{cm} 2)$ & 1.82 & 1.49 & 2.32 & 1.26 & 4.74 & $*$ & 0.36 \\
\hline Mean velocity of $X$-axis $(\mathrm{cm} / \mathrm{sec})$ & 0.77 & 0.27 & 1.04 & 0.39 & 10.60 & $*$ & 0.81 \\
\hline Mean velocity of $Y$-axis $(\mathrm{cm} / \mathrm{sec})$ & 0.59 & 0.20 & 0.90 & 0.38 & 13.19 & $*$ & 1.00 \\
\hline Root mean square of sway velocity $(\mathrm{cm} / \mathrm{sec})$ & 1.62 & 0.54 & 2.28 & 0.87 & 12.16 & $*$ & 0.93 \\
\hline Standard deviation of $\mathrm{X}$-axis velocity $(\mathrm{cm} / \mathrm{sec})$ & 1.28 & 0.45 & 1.72 & 0.64 & 10.51 & $*$ & 0.80 \\
\hline Standard deviation of $\mathrm{Y}$-axis velocity $(\mathrm{cm} / \mathrm{sec})$ & 0.98 & 0.34 & 1.47 & 0.63 & 13.00 & $*$ & 0.99 \\
\hline Ratio of A domain for power spectrum of $\mathrm{X}$-axis $(\%)$ & 26.68 & 6.25 & 26.47 & 6.45 & 0.45 & & 0.03 \\
\hline Ratio of C domain for power spectrum of $X$-axis (\%) & 13.79 & 3.65 & 14.10 & 4.41 & 0.99 & & 0.08 \\
\hline Ratio of $C$ domain for power spectrum of $Y$-axis (\%) & 16.56 & 4.73 & 17.23 & 5.44 & 1.73 & & 0.13 \\
\hline Ratio of A domain for power spectrum of R-axis (\%) & 24.40 & 5.68 & 21.72 & 5.84 & 6.07 & $*$ & 0.46 \\
\hline Ratio of C domain for power spectrum of R-axis (\%) & 16.76 & 4.05 & 18.92 & 5.52 & 5.90 & $*$ & 0.45 \\
\hline Fasio of Adomain sor power spectrum of $X$-wis velocity (\%6) & 4.13 & 1.48 & 4.42 & 1.66 & 2.43 & * & 0.19 \\
\hline Fastio of $C$ domain tor powar spectrum of $X$-axis velocity (\%) & 25.49 & 3.69 & 25.25 & 4.98 & 0.75 & & 0.06 \\
\hline Festio of $C$ domin for power spectrum of $Y$-sics velocity (\%) & 27.88 & 4.08 & 30.43 & 6.29 & 6.41 & $*$ & 0.49 \\
\hline 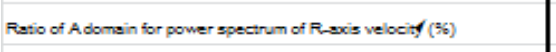 & 8.90 & 1.79 & 8.98 & 2.08 & 0.55 & & 0.04 \\
\hline 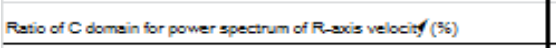 & 44.44 & 4.31 & 45.41 & 6.13 & 2.42 & $*$ & 0.19 \\
\hline Mean vector length of A direction sway $(\mathrm{cm})$ & 0.67 & 0.28 & 0.73 & 0.25 & 2.72 & $*$ & 0.21 \\
\hline Mean vector length of $\mathrm{C}$ direction sway $(\mathrm{cm})$ & 0.55 & 0.22 & 0.69 & 0.23 & 7.64 & $*$ & 0.58 \\
\hline Mean vector length of $E$ direction sway $(\mathrm{cm})$ & 0.66 & 0.26 & 0.74 & 0.26 & 4.03 & $*$ & 0.31 \\
\hline Mean vector length of $\mathrm{G}$ direction sway $(\mathrm{cm})$ & 0.55 & 0.21 & 0.68 & 0.22 & 7.79 & * & 0.60 \\
\hline Mean vector length of A direction velocity ( $\mathrm{cm} / \mathrm{sec}$ ) & 0.91 & 0.31 & 1.35 & 0.59 & 12.52 & $*$ & 0.96 \\
\hline Mean vector length of $C$ direction velocity $(\mathrm{cm} / \mathrm{sec})$ & 1.21 & 0.40 & 1.62 & 0.61 & 10.57 & $*$ & 0.81 \\
\hline Mean vector length of E direction velocity ( $\mathrm{cm} / \mathrm{sec}$ ) & 0.93 & 0.32 & 1.39 & 0.63 & 12.37 & $*$ & 0.95 \\
\hline Mean vector length of $\mathrm{G}$ direction velocity ( $\mathrm{cm} / \mathrm{sec})$ & 1.21 & 0.40 & 1.62 & 0.63 & 10.37 & $*$ & 0.79 \\
\hline
\end{tabular}

note) $\alpha^{\prime}=0.05 / 30, * \mathrm{p}<\alpha^{\prime}$

Table 2. Correlation coefficients between parameters for young adults

\begin{tabular}{|c|c|c|c|c|c|c|c|c|c|c|c|c|c|c|c|c|c|c|c|c|c|c|c|c|c|c|c|c|c|c|}
\hline \multirow{2}{*}{ 1/Mean path lenghth (crisec) } & 1 & 2 & 3 & 4 & 5 & 6 & 7 & 8 & 9 & 10 & 11 & 12 & 13 & 14 & 15 & 16 & 17 & 18 & 19 & 20 & 21 & 22 & 23 & 24 & 25 & 26 & 27 & 28 & 29 & 30 \\
\hline & & & & & & & & & & & & & & & & & & & & & & & & & & & & & & \\
\hline 2 Rootmean square (cm) & 0.58 & & & & & & & & & & & & & & & & & & & & & & & & & & & & & \\
\hline 3 Rot mean square of X-axis (cm) & 056 & 0.76 & & & & & & & & & & & & & & & & & & & & & & & & & & & & \\
\hline 4 Root mean square of Y-axis (cm) & $0.50 *$ & $0.99^{*}$ & 0.48 & & & & & & & & & & & & & & & & & & & & & & & & & & & \\
\hline 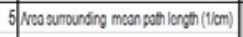 & -0.14 & -0.75 & $-0,000$ & -0.68 & & & & & & & & & & & & & & & & & & & & & & & & & & \\
\hline 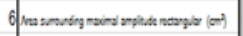 & $0.14=$ & 087 & 0.78 & $0.7 n$ & -051 & & & & & & & & & & & & & & & & & & & & & & & & & \\
\hline 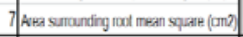 & 062. & 0.08 & 074 & 0.89 & -10000 & 099. & & & & & & & & & & & & & & & & & & & & & & & & \\
\hline 8 Mean velocity of $X$-axis (cmsec) & 0.97 * & 057 & 0.58 & 0.47 & -0.15 & 0.72 & 0.61 & & & & & & & & & & & & & & & & & & & & & & & \\
\hline 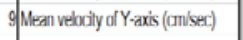 & 088 & 0000 & 053 & $0.53 \times$ & $-1210 \times 0$ & $074 \times 0$ & $062 \times$ & 081 & & & & & & & & & & & & & & & & & & & & & & \\
\hline 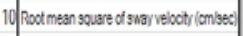 & 0.99 * & 061 & 059 & 052 & 0.10 & 076 & 0.64 & 0.97 * & 092 & & & & & & & & & & & & & & & & & & & & & \\
\hline 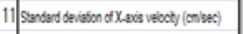 & 0.058 & 057 & 0.58 & 0.4 & -0.15 & 0.72 & 0.51 & 100 & 081 & $0.91 *$ & & & & & & & & & & & & & & & & & & & & \\
\hline 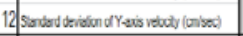 & 092 & 000 & 0.54 & $0.54 *$ & $-122=0$ & $0.74=0$ & $0.62 *$ & 081 & 100 & 092 & 0.81 & & & & & & & & & & & & & & & & & & & \\
\hline 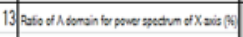 & -034 & 0.17 & $0.38=$ & 0,0 & $-0,18$ & 009 & 0.12 & -031 & -021 & -032 & -037 & -020 & & & & & & & & & & & & & & & & & & \\
\hline 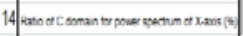 & 016 & 000 & -0.06 & 000 & 018 & 000 & 003 & 013 & 010 & 012 & 012 & 000 & -038 & & & & & & & & & & & & & & & & & \\
\hline 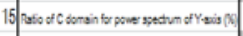 & 0.11 & 0.14 & -0.04 & 022 & $-0,01$ & 0.07 & 0.12 & 0.08 & 0.07 & 0.08 & 0.08 & 0.07 & -0.15 & 0.14 & & & & & & & & & & & & & & & & \\
\hline 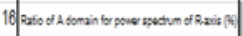 & -032 & $0.40=$ & $0.14 x$ & $0.44 \times$ & -0590 & $0.18=0$ & $0.33=$ & -031 & -024 & -030 & -031 & -023 & 049 & -0.14 & $-0,03$ & & & & & & & & & & & & & & & \\
\hline 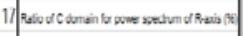 & 0.37 & 022 * & 0.10 * & $0.19 *$ & 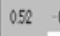 & $0.12 *$ & 0.110 & 034 & 025 & 032 & 034 & 024 & .055 & 0.41 & 020 & $0 \infty$ & & & & & & & & & & & & & & \\
\hline 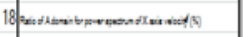 & -033 & 0.13 & 027 & 0,8 & -0.45 & 009 & 0.09 & -031 & -0.18 & -0.31 & -037 & -0.17 & 084 & -024 & -0.16 & 0.48 & -0.54 & & & & & & & & & & & & & \\
\hline 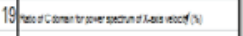 & 020 & -006 & -0.08 & -0.04 & 024 & $-0,05$ & -0.07 & 0.14 & 0.12 & 0.14 & 0.14 & 0.12 & -0.18 & 0.45 & 022 & -024 & 052 & $-0,31$ & & & & & & & & & & & & \\
\hline 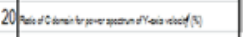 & 026 & $-0,08$ & -0.08 & -0.06 & 031 & $-0,03$. & -0.06 & 021 & 0.14 & 0.19 & 021 & 0.12 & -028 & 033 & 038 & -030 & $059-$ & -035 & 055 & & & & & & & & & & & \\
\hline 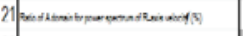 & $-0,06$ & 012 & 008 & 013 & -028 & 013 & $O C B$ & -004 & 001 & 001 & -001 & 000 & 009 & $-0 x$ & -010 & 014 & $\rightarrow n 0$ & $016-$ & -034 & -029 & & & & & & & & & & \\
\hline 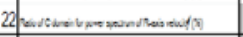 & 028 & -0.18 & -0.12 & -0.11 & 0.41 & -0.11 & -0.11 & 024 & 0.16 & 021 & 028 & 0.14 & -025 & 0.47 & 026 & $-\infty 36$ & $0.65-1+1-1-1-1$ & -0.490 & 0.70 & 062 & -052 & & & & & & & & & \\
\hline 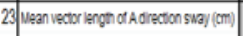 & 0.45 & 087 & 0,09 & 0.91 & $-0,66$ & 073 & 083 & 0.430 & $0.51 x$ & 0.99 & 0.43 & $0.52 \times$ & 0006 & 001 & 022 & $0.38 \mathrm{x}-1$ & $-0.18 \times 0$ & 0,070 & 0.000 & $-0,108$ & 0.12 & -0.14 & & & & & & & & \\
\hline 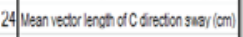 & 0.40 & 0.67 & 000 & 0.4 & .056 & 0.72 & 0.67 & 0.49 & 0.46 & 0.51 & 050 & 0.47 & $0.96 *$ & -0.65 & -0.00 & 0.14 * & $020=0$ & $0.31 *$ & -0.10 & .009 & 0.10 & $0.17 *$ & 0.46 & & & & & & & \\
\hline 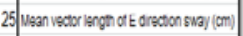 & 0,13 & $0.96 \%$ & 0.9 & 091 & -060 & 0,1 & 082 & 0.41 & 0.15 & 0.5 & 0.12 & 0.15 & 0.07 & 0.04 & 0.14 & $0.16 x-1-3(x-1)$ & $-121 \times 0$ & 0.08 - & -0.08 & $-0,08$ & 0.15 & -0.13 & 0.73 & 0.86 & & & & & & \\
\hline 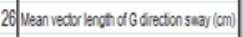 & 0.52 & 006 & $0 \otimes 8$ & 0.4 & -055 & 0.07 & 005 & 0.54 & 0.49 & 0.54 & 0.54 & 0.49 & 0.32 * & -0.05 & -0.08 & $0.12 *-1$ & $-0.15 * 0$ & $022-$ & -0.07 & -0.10 & 0.00 & -0.10 & 040 & 0.71 & 0.42 & & & & & \\
\hline 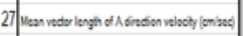 & 098 & 0.58 & 0.52 & $0.51 \times$ & $-120 \times 0$ & $0.71 \div 0$ & $0.60 \times$ & 0.78 & 096 & 099 & 0.78 & 0.96 & -200 & 000 & 0.06 & -223 & $022-4-3-3-10$ & -0.170 & 0.11 & 0.10 & 0.00 & 0.14 & 050 & 0.15 & 0.13 & 0.18 & & & & \\
\hline 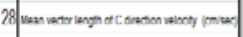 & 0.5 . & 0.58 & 0.58 & 047 & -016 & on & 061 & 097 & OB2 & 098 & 098 & 081 & -0.35 & 012 & 007 & -030 & $0.38-$ & -0.58 & 014 & on & -002 & 023 & 044 & 050 & 042 & 054 & 079 & & & \\
\hline 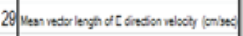 & 098 & 0.59 . & 0.51 & 0.58 * & $-122 \cdot 0$ & $0.73 \cdot 0$ & 0.61 • & 0.7 & 0.96 & 098 & 0.7 & 0.97 & -0.18 & 0.11 & 0.04 & -221 & $028-4-3-1-1-10$ & -0.15 & 0.12 & 0.10 & 0,02 & 0.14 & 0.52 . & 0.4 & 0.55 & 0.47 & 098 & 0.78 & & \\
\hline 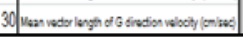 & $0.05 \mathrm{x}$ & 0.58 & 0.56 & 0.48 & -0.16 & 0.71 & 0.61 & 0.08 & 0.82 & $0.96 \times 0$ & & 0.81 & -0.37 & 0.13 & 0.08 & -030 & $0.84-$ & $-0.37 \quad 0$ & 0.13 & 0.19 & $-0,012$ & 0.2 & 0.45 & 0.48 & 0.43 & 0.53 & 0.80 & 0.95 & 0.7 & \\
\hline
\end{tabular}


Table 3. Correlation coefficients between parameters for the elderly

\begin{tabular}{|c|c|c|c|c|c|c|c|c|c|c|c|c|c|c|c|c|c|c|c|c|c|c|c|c|c|c|c|c|c|c|}
\hline & 1 & 2 & 3 & 4 & 5 & 6 & 1 & 8 & 9 & 10 & 11 & 12 & 13 & 14 & 15 & 16 & 17 & 18 & 19 & 20 & 21 & 22 & 23 & 24 & 25 & 26 & 21 & 28 & 29 & 30 \\
\hline \multicolumn{31}{|l|}{ 1| Mean path length (cm/sec) } \\
\hline 2 Rool mean square (cm) & 0.51 & & & & & & & & & & & & & & & & & & & & & & & & & & & & & \\
\hline 3 Root mean square of X-axis (cm) & 0.55 & 080 & & & & & & & & & & & & & & & & & & & & & & & & & & & & \\
\hline 4 Root mean square of $Y$ axis (cm) & 0.37 & 0.90 & 0.45 & & & & & & & & & & & & & & & & & & & & & & & & & & & \\
\hline 5 Mra surounang maen pas lengen (1/cm) & 0.00 & -0.72 & -058 & -0.65 & & & & & & & & & & & & & & & & & & & & & & & & & & \\
\hline 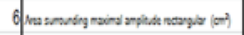 & 0.63 & 0.85 & 0.78 & 0.72 & -058 & & & & & & & & & & & & & & & & & & & & & & & & & \\
\hline 7 Avea sumunfing roul mean square (um) & 050 & 098 * & $0 \pi$ & 0.90 & -068 & 088 & & & & & & & & & & & & & & & & & & & & & & & & \\
\hline : Mcan velocity of $X$ axis (cm/scc) & 092 & 0.59 & 0.65 & 0.42 & 0.11 & 067 & 057 & & & & & & & & & & & & & & & & & & & & & & & \\
\hline of Mean velocity of $Y$-axis (cm/sec) & 0.96 . & 0.51 & 053 & 038 & -0.04 & 0.68 & 0.49 & $0 \& 8$ & & & & & & & & & & & & & & & & & & & & & & \\
\hline 10. Rootmean squere of swaj veloct/ (cmssec) & 098 & 0.58 & 0.63 & 0.42 & $-0,09$ & 0.70 & 0.56 & 0.96 & $0.95 \mathrm{x}$ & & & & & & & & & & & & & & & & & & & & & \\
\hline 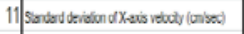 & 092 & 0.59 & 0.00 & 0.42 & -0.12 & 000 & 057 & 1.00 & 083 & 090 & & & & & & & & & & & & & & & & & & & & \\
\hline 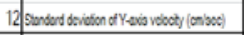 & 0.95 . & 0.51 & 0.54 & 038 & -0.05 & 064 & 0.40 & 0.83 & 1.00 & $0.95^{\circ}$ & 0.88 & & & & & & & & & & & & & & & & & & & \\
\hline 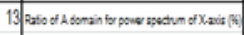 & -0.42 & 0,0 & 020 & -0.09 & -039 & $-0,13$ & 0.07 & $-0,4$ & $-0,35$ & -0.41 & $-0,4$ & $-0.35 \times$ & & & & & & & & & & & & & & & & & & \\
\hline 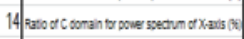 & $0.40 *$ & 0.07 & 0.08 & 0.04 & 025 & 000 & 0.07 & $0.32 *$ & 038 & 035 & 031 & 087 & -0.47 & & & & & & & & & & & & & & & & & \\
\hline 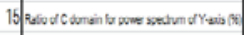 & $0.50=$ & 0.15 & 0.10 & 0.14 & 0.25 * & 0.16 & 0.16 & $0.39=$ & $0.44 *$ & $0.42 *$ & $0.30=$ & $0.40 *$ & 034 * & 0.96 * & & & & & & & & & & & & & & & & \\
\hline 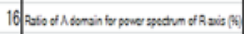 & $-0.32=$ & 0.16 & $-0,08$ & 028 & -0.99 & -0.02 & 0.16 & $-0.13 \times$ & $-0.50 \times$ & $-0.51 \times$ & $-0.18 \times$ & $-0.50 \times$ & 051 & -027 & -037 & & & & & & & & & & & & & & & \\
\hline 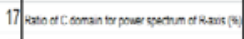 & 070. & -001 & 004 & $-0,0$ & 052 & 011 & 000 & 0.57. & 064 . & 0 62. & 056 . & 0.03. & -0.55 & 0.55 * & 065 . & -1060 & & & & & & & & & & & & & & \\
\hline 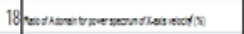 & .030 & 000 & 0.15 & -0.00 & 0.37 & .002 & 0.02 & -0.40 & -0.00 & $0 x 9$ & -0.40 & .090 & 000 & -034 & $0.4 *$ & 0.41 & .050 & & & & & & & & & & & & & \\
\hline 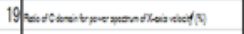 & $0.15 \times$ & -0.01 & -0.02 & 0.01 & 034 & 007 & 0.00 & $0.32=$ & $0.41 *$ & $0.37 \times$ & $0.31 \times$ & $0.40 \times$ & -031 & $0.58 *$ & $0.15=$ & -030 & 0.62 & -0.11 & & & & & & & & & & & & \\
\hline 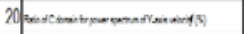 & 051 . & 000 & 008 & 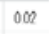 & 038 & 011 & 000 & 0.37. & 042. & 040. & 036. & 041 * & -031 & 041 & 070. & -037 & 071 * & $-0,39$ & 000 & & & & & & & & & & & \\
\hline 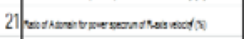 & $022=$ & 0.10 & 0.10 & 0.00 & .036 & 0.17 & 0.10 & -0.10 & 0.10 & -0.10 & -0.10 & -0.15 & 0.16 & -026 & $0.4 *$ & 020 & $0.10 *$ & 020 & -0.40 & $0.19 *$ & & & & & & & & & & \\
\hline 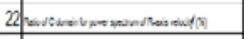 & 0.50 . & -0.01 & 0.01 & $-0,02$ & 0.45 & 0.01 & -0.01 & $0.40^{\circ}$ & 0.43 . & $0.41^{\circ}$ & 0.38 。 & 0.41 . & -036 & 054 & 0.58 * & -0.44 & $0.73^{\circ}$ & -0.18 & 0.71 & $0.74^{*}$ & $-0,60$ & & & & & & & & & \\
\hline 23 Magn vector lenghn or A direction sway (cm) & 038 & 0.85 & 0.50 & 0.90 & -0.59 & 0.68 & 0.96 & 0.44 & 0.39 & 0.43 & 0.43 & 0,39 & -0.01 & 0.05 & 0.13 & 0.18 & 000 & -0.06 & 0.00 & 004 & 0,003 & 0.01 & & & & & & & & \\
\hline 24 Nean vedtor lench di C direction swaj (cm) & 0.40 & 071 & 0.87 & 0.42 & -0.51 & 0.67 & 0.68 & 0.55 & 0.45 & 0.52 & 0.56 & 0.44 & 021 & 0.07 & 0.09 & -0.05 & 0018 & 0.17 & -0.08 & 0.07 & 0.10 & -0.01 & 0.47 & & & & & & & \\
\hline 25 Mean vedtor longh of E dircdion onoy (cm) & 034 & 082 & 0.4 & 0.90 & -0.60 & 065 & 082 & 0.37 & 036 & 038 & 0.37 & 0.35 & $0,0 B$ & 0.00 & 0.10 & 027 & -0.08 & -0.08 & -0.01 & 0.01 & 0.06 & -0.08 & 0.72 & 039 & & & & & & \\
\hline 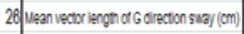 & 0.53 & 0.70 & 0.87 & 0.42 & -0.00 & 0.57 & 0.67 & 060 & 050 & 0.58 & 0.60 & 0.50 & 0.17 & 0.07 & 0.10 & -0.11 & 0018 & 0.12 & 0.00 & $0.11 \times$ & 0.04 & 0.06 & 0.47 & 069 & 0.41 & & & & & \\
\hline 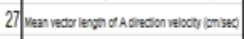 & 0.94 * & 0.50 & 0.54 & 0.87 & -0.04 & 062 & 0.48 & 0.81 & $0.98 *$ & 0.93 * & 0.81 & $0.98 *$ & -082 & $0.35 *$ & $0.43=$ & $-0.50 *$ & $0.62 *$ & -028 & 0.41 * & 0.42 * & $-1.19 *$ & $=0.42=$ & 038 & 0.44 & 0.34 & 0.50 & & & & \\
\hline 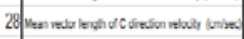 & 091 & 057 & 0.64 & 030 & -0.10 & 0.64 & 0.54 & 097 & 000 & 0.94 & 0.97 & $0 Q 8$ & -0.42 & $0.29 *$ & $0.30 *$ & $-0.50 *$ & * $0.57 *$ & -0.42 & 0.31 * & $0.39=$ & $=0.16$ & $0.41 *$ & 0.42 & 0,54 & 0,35 & 0.80 & 001 & & & \\
\hline 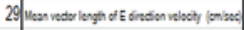 & $0.94=$ & 0.98 & 0.9 & 035 & -0.01 & 058 & 0.16 & $0 \$ 8$ & $0.98 \times$ & $0.92 \times$ & 0.78 & 097 & $-1031=$ & $0.38 \mathrm{x}$ & $0.14 \times$ & $-0.18=$ & $0.55=$ & $-1.31=$ & $0.11 \times$ & $0.13 *$ & -021 & $0.14=$ & 037 & 0.90 & 034 & 0.46 & $0.85 \times$ & 080 & & \\
\hline 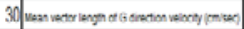 & 091 & 0.58 & 0,03 & 041 & -011 & О६Б & 056 & 097 & 083 & 0.94 & 0.97 & 083 & -044 & 032. & 039. & -1018. & $.057^{\circ}$ & -041 & 0.32 . & 038. & -013 & 040. & 042 & 053 & 037 & 0.58 & 0.81 & 0.56 & 080 & \\
\hline
\end{tabular}

Table 4. Frequency outside the young adults' mean+1-3SD range

\begin{tabular}{|c|c|c|c|c|c|c|c|c|}
\hline & & & \multicolumn{6}{|c|}{ elderly } \\
\hline & \multicolumn{2}{|c|}{ young adults } & \multicolumn{2}{|c|}{$\mathrm{M} \pm 1 \mathrm{SD}$} & \multicolumn{2}{|c|}{$\mathrm{M} \pm 2 \mathrm{SD}$} & \multicolumn{2}{|c|}{$M \pm 3 S D$} \\
\hline & $\mathrm{M}$ & $\mathrm{SD}$ & frequency & $(\%)$ & frequency & $(\%)$ & frequency & $(\%)$ \\
\hline Mean path length $(\mathrm{cm} / \mathrm{sec})$ & 1.21 & 0.41 & 151 & 48.6 & 75 & 24.1 & 36 & 11.6 \\
\hline Root mean square $(\mathrm{cm})$ & 0.72 & 0.24 & 81 & 26.0 & 20 & 6.4 & 2 & 0.6 \\
\hline Root mean square of $X$-axis $(\mathrm{cm})$ & 0.44 & 0.15 & 107 & 34.4 & 45 & 14.5 & 10 & 3.2 \\
\hline Root mean square of $Y$-axis $(\mathrm{cm})$ & 0.56 & 0.22 & 53 & 17.0 & 10 & 3.2 & 3 & 1.0 \\
\hline Area surrounding mean path length $(1 / \mathrm{cm})$ & 26.88 & 10.06 & 34 & 10.9 & 9 & 2.9 & 0 & 0.0 \\
\hline Area surrounding maximal ampitude rectangular $\left(\mathrm{cm}^{2}\right)$ & 7.23 & 5.44 & 89 & 28.6 & 39 & 12.5 & 12 & 3.9 \\
\hline Area surrounding root mean square $(\mathrm{cm} 2)$ & 1.82 & 1.49 & 51 & 16.4 & 16 & 5.1 & 2 & 0.6 \\
\hline Mean velocity of $X$-axis $(\mathrm{cm} / \mathrm{sec})$ & 0.77 & 0.27 & 130 & 41.8 & 63 & 20.3 & 24 & 7.7 \\
\hline Mean velocity of $Y$-axis $(\mathrm{cm} / \mathrm{sec})$ & 0.59 & 0.20 & 163 & 52.4 & 91 & 29.3 & 48 & 15.4 \\
\hline Root mean square of sway velocity (cm/sec) & 1.62 & 0.54 & 145 & 46.6 & 78 & 25.1 & 35 & 11.3 \\
\hline Standard deviation of $\mathrm{X}$-axis velocity $(\mathrm{cm} / \mathrm{sec})$ & 1.28 & 0.45 & 130 & 41.8 & 66 & 21.2 & 24 & 7.7 \\
\hline Standard deviation of $Y$-axis velocity $(\mathrm{cm} / \mathrm{sec})$ & 0.98 & 0.34 & 158 & 50.8 & 89 & 28.6 & 46 & 14.8 \\
\hline Ratio of A domain for power spectrum of $\mathrm{X}$-axis (\%) & 26.68 & 6.25 & 52 & 16.7 & 4 & 1.3 & 1 & 0.3 \\
\hline Ratio of $\mathrm{C}$ domain for power spectrum of $\mathrm{X}$-axis (\%) & 13.79 & 3.65 & 67 & 21.5 & 24 & 7.7 & 7 & 2.3 \\
\hline Ratio of $\mathrm{C}$ domain for power spectrum of $\mathrm{Y}$-axis (\%) & 16.56 & 4.73 & 65 & 20.9 & 16 & 5.1 & 6 & 1.9 \\
\hline Ratio of A domain for power spectrum of R-axis (\%) & 24.40 & 5.68 & 21 & 6.8 & 6 & 1.9 & 1 & 0.3 \\
\hline Ratio of C domain for power spectrum of R-axis (\%) & 16.76 & 4.05 & 91 & 29.3 & 41 & 13.2 & 12 & 3.9 \\
\hline Ratio of A domain for power spectrum of X-axis velocity (\%) & 4.13 & 1.48 & 52 & 16.7 & 19 & 6.1 & 5 & 1.6 \\
\hline Ratio of C domain for power spectrum of $X$-axis velocity (\%) & 25.49 & 3.69 & 63 & 20.3 & 22 & 7.1 & 10 & 3.2 \\
\hline Ratio of $\mathrm{C}$ domain for power spectrum of $\mathrm{Y}$-axis velocity (\%) & 27.88 & 4.08 & 117 & 37.6 & 49 & 15.8 & 21 & 6.8 \\
\hline Ratio of A domain for power spectrum of R-axis velocity (\%) & 8.90 & 1.79 & 63 & 20.3 & 22 & 7.1 & 6 & 1.9 \\
\hline Ratio of $\mathrm{C}$ domain for power spectrum of $\mathrm{R}$-axis velocity (\%) & 44.44 & 4.31 & 90 & 28.9 & 34 & 10.9 & 9 & 2.9 \\
\hline Mean vector length of A direction sway $(\mathrm{cm})$ & 0.67 & 0.28 & 49 & 15.8 & 11 & 3.5 & 3 & 1.0 \\
\hline Mean vector length of $\mathrm{C}$ direction sway $(\mathrm{cm})$ & 0.55 & 0.22 & 99 & 31.8 & 28 & 9.0 & 9 & 2.9 \\
\hline Mean vector length of $\mathrm{E}$ direction sway $(\mathrm{cm})$ & 0.66 & 0.26 & 58 & 18.6 & 22 & 7.1 & 4 & 1.3 \\
\hline Mean vector length of $\mathrm{G}$ direction sway $(\mathrm{cm})$ & 0.55 & 0.21 & 98 & 31.5 & 32 & 10.3 & 10 & 3.2 \\
\hline Mean vector length of A direction velocity ( $\mathrm{cm} / \mathrm{sec})$ & 0.91 & 0.31 & 153 & 49.2 & 87 & 28.0 & 48 & 15.4 \\
\hline Mean vector length of $\mathrm{C}$ direction velocity $(\mathrm{cm} / \mathrm{sec})$ & 1.21 & 0.40 & 137 & 44.1 & 64 & 20.6 & 31 & 10.0 \\
\hline Mean vector length of E direction velocity ( $\mathrm{cm} / \mathrm{sec}$ ) & 0.93 & 0.32 & 155 & 49.8 & 83 & 26.7 & 45 & 14.5 \\
\hline Mean vector length of $\mathrm{G}$ direction velocity $(\mathrm{cm} / \mathrm{sec})$ & 1.21 & 0.40 & 128 & 41.2 & 66 & 21.2 & 26 & 8.4 \\
\hline
\end{tabular}




\section{Discussion}

Demura et al. [5] and Kitabayashi et al. [3] compiled 114 parameters used so far in many studies and examined the trial-to-trial and day-to-day reliabilities, interrelationships among parameters, and gender differences of these parameters. They reported that the characteristics of COP sway can be synthetically understood using only 30 parameters. The body sway characteristics of the elderly were compared with those of the young adults using the above 30 parameters in the present study. The elderly showed large values for 25 parameters and not for 5 parameters. Considerable differences were observed in the mean path length regarding sway size, root mean square of $\mathrm{x}$-axis for right/left sway size, velocity, and vector velocity parameters regarding sway velocity. Body sway in the elderly is thus considered to be larger and quick. According to Rogind et al. [6] and Brooke-Wavell et al. (2002), the large decline in body sway in the elderly can be attributed to changes in the following factors: labyrinth and proprioception systems with age, leg strength needed to maintain posture, and adjustment ability of leg strength related to moving body weight. The abovestated parameters are related to sway size and velocity. These parameters in the elderly were remarkably larger than those in the young adults. Therefore, these are considered effective parameters reflecting body sway characteristics and the functional decline in posture maintenance in the elderly. In addition, on comparing correlations in the young adults and the elderly, we found that many parameters were similar in the two groups, but correlations of parameters related to velocity, power spectrum, and vector velocity were significantly greater in the elderly than in the young adults. When evaluating the body sway of healthy people, it is necessary to consider individual differences and to synthetically evaluate COP sway using plural parameters [7] (Demura et al., 2002). The relationships between velocity parameters used to evaluate the details of body sway and the other parameters are stronger in the elderly than in the young adults. Therefore, the body sway characteristics of the elderly can be evaluated synthetically and simply using a small number of velocity parameters.

On the other hand, Nakagawa et al. [8] and Hattori et al. [9] demonstrated that individual differences in the body sway of the elderly can be large. Mizuta \& Miyata [10] reported that it is necessary to consider the distribution characteristics of COP parameters when evaluating sway in the elderly. In short, when selecting effective parameters for evaluating body sway, it is necessary to consider individual differences.

Hence, we examined the distribution characteristics of the parameters among elderly participants on the basis of the values for the young adults. When the data followed a normal distribution, $99.74 \%$ of the data fell within a range of mean \pm 3 SD. Thus, data outside this range may be judged as abnormal values [11]. Tokita et al. [7] examined sway characteristics in disabled persons with abnormal sway using the range of mean \pm 2 SD or more as the basis. Moreover, in that study, the distribution for the elderly was based on the reference range for young adults. Therefore, approximately $80 \%-90 \%$ of values for the elderly were included within their mean \pm 1 SD. However, for parameters related to body sway size and velocity, approximately half (40\%-50\%) fell outside the reference range. In addition, data for almost all parameters were within the range of mean $\pm 3 \mathrm{SD}$, but those for parameters related to body sway size and velocity, $\geq 10 \%$ of the data in the elderly were outside this range. The sway characteristics of the elderly were found to be particularly represented by parameters in the front/back direction even among velocity parameters. These reflect the body sway characteristics of the elderly who repeat delicate and quick sways. From the above findings, we conclude that the parameters noted above may be effective for evaluating body sway in the elderly.

\section{Conclusion}

In conclusion, the following parameters may be useful to evaluate body sway characteristics of the elderly adequately and simple: sway size, sway velocity, and from a viewpoint of a direction, sway size in the right/left direction and sway velocity in the front/back direction.

\section{References}

[1] Fujiwara, K., \& Ikegami, H. (1984) Chronological change in frequency component of body sway in up right stance. Japanese Journal of Human Posture, 2, 81-88.

[2] Hirasawa, K., Starkes, J., \& Takahashi, T. (1972) The influence of age on variability of postural sway. Japanese Journal of Human Posture, 11, 137-146. Japan Society for Equilibrium Research. (1994) A Fact of Equilibrium Research. Nanzando, Japan. (In Japanaese).

[3] Kitabayashi, T., Demura, S., \& Noda, M. (2003) Examination of the factor structure of center of foot pressure movement and crossvalidity. Journal of Physiological Anthropology and Applied Human Science, 22, 265-272.

[4] Kitabayashi, T., Demura, S., Yamaji, S., \& Kaoru, I. (2002) Gender differences and relationships between physic and parameters evaluating the body center of pressure in static standing posture. Equilibrium Research, 61, 16-17.

[5] Demura, S., Yamaji, S., Noda, M., Kitabayashi, T., \& Nagasawa, Y. (2001) Examination of parameters evaluating the center of foot pressure in static standing posture from viewpoints of trial-to-trial reliability and interrelationships among parameters. Equilibrium Research, 60, 44-55.

[6] Rogind, H., Lykkegaard, J. J., Bliddal, H., \& Danneskiold-Samsoe, B. (2003) Postural sway in normal subjects aged 20-70 years. Clinical Physiology and Functional Imaging, 23, 171-176.

[7] Tokita, T., Tokumasu, K., Imaoka, K., Murase, H., \& Fukuhara, M. (2001) Classification of Stabilograms in Healthy Subjects Using Neural Network. Equilibrium Research, 60,181-187.

[8] Nakagawa, H., Ohashi, N., Watanabe, Y., \& Mizukoshi, K. (1993) The contribution of proprioception to posture control in normal subjects. Acta Oto-Larynogologica, 504, 112-116.

[9] Hattori, K., Starkes, J., \& Takahashi, T. (1992) The influence of age on variability of postural sway during the daytime. Japanese Journal of Human Posture, 11, 137-146.

[10] Mizuta, K., \& Miyata, H. (1993) Standing posture of human. Sogo Rehabilitation, 21, 985-990.

[11] Demura, S., \& Kitabayashi, T. (2006) Comparison of power spectrum characteristics of body sway during a static upright standing posture in healthy elderly people and young adults. Perceptual and Motor Skills, 102, 467-476.

[12] Goldie, P. A, Bach, T. M, \& Evans, O. M. (1989) Force platform measures for evaluating postural control: reliability and validity. American Congress of Rehabilitation Medicine, 70, 510-517.

[13] Njiokiktjien, C., \& Folkerts, J. F. (1971) Displacement of the body's center of gravity at galvanic stimulation of the labyrinth. Confinia Neurologica, 33, 46-54. 
[14] Pyykko I (2000). Evaluation of postural stability. Equilibrium Res 59:401-407.
[15] Shimada, H., Obuchi, S., Kamide, N., Shiba Y., Okamoto, M., \& Kakurai, S. (2003). Relationship with dynamic balance function during standing and walking. American Journal of Physical Medicine and Rehabilitation, 82, 511-516. 